\title{
Ascites: a loadstar for the diagnosis and management of an intracranial tumor
}

\author{
Hayriye Hızarcıoğlu Gülşenen ${ }^{1,2, *}$, Adem Kurtuluş̧, ${ }^{3, *}$ \\ Departments of ${ }^{1}$ Pediatric Gastroenterology and ${ }^{3}$ Neurosurgery, University of Health Sciences Ankara Health Research and Application \\ Center, Ankara; ${ }^{2}$ Department of Pediatric Gastroenterology, Hacettepe University Faculty of Medicine, Ankara, Turkey.
}

\begin{abstract}
Background. Ascites is defined as abnormal fluid retention in the peritoneal cavity and it can be encountered at any age including fetal life. Ascites mostly results from cirrhosis, chronic renal disease and heart failure in childhood. However, there are various reasons for cirrhotic and non-cirrhotic ascites in the pediatric age. Cerebrospinal fluid ascites is one of the rarest.

Case. A 3.5- year- old Sudanese boy who underwent right-sided ventriculoperitoneal shunt surgery for hydrocephalus 10 months ago was admitted to the Neurosurgery Intensive Care Unit for intracranial tumor surgery. He had neurologic deterioration and ascites accumulation for the last 4 months. He was consulted with the pediatric gastroenterologist to exclude the reasons causing ascites after admission. No chronic liver, renal or heart disease was shown. The gross appearance of ascitic fluid was so clear that it resembled the cerebrospinal fluid and laboratory analysis results were compatible with transudate. The magnetic resonance imaging identified a mass in the left lateral ventricle. From the pediatrician's perspective, overproduction of cerebrospinal fluid from a tumor was assumed and shunt exclusion was proposed to alleviate ascites. After the externalization of the stunt and external ventricular device implementation, no further ascites occurred. The patient had a successful tumor excision and discharged after gaining oral feeding ability and sufficient weight gain.
\end{abstract}

Conclusion. In case of intractable ascites occurrence after a ventriculoperitoneal shunt placement, a pediatrician should consider etiologies resulting in imbalance of absorption and secretion function of cerebrospinal fluid.

Key words: ascites, ventriculoperitoneal shunt, choroid plexus papilloma.

Peritoneal fluid is the product of ultrafiltration activity of the peritoneum. ${ }^{1}$ In healthy individuals, visceral and parietal layers of the peritoneum are flushed with a small volume of peritoneal fluid, typically $5-20 \mathrm{~mL}$ or up to $100 \mathrm{ml}$ at most. ${ }^{1,2}$ Ascites is the abnormal accumulation of peritoneal fluid secondary to the imbalance between peritoneal secretion and absorption functions. ${ }^{2}$ Cirrhosis is the most common cause of pediatric ascites. However, non-cirrhotic factors should be reviewed in

\section{Hayriye Hızarcıoğlu Gülşen}

hayriyegulsen@hacettepe.edu.tr

* Both authors contributed equally.

Received 8th April 2020, revised 4th May 2020, accepted 4th May 2020. patients with ascites and good functioning livers. Here, we present a patient referred to our hospital with a symptom that accelerated the diagnosis and treatment of an intracranial tumor; resistant ascites.

\section{Case Report}

A 3.5- year- old Sudanese boy was admitted to our Neurosurgery Intensive Care Unit for the management of his intracranial tumor. He was consulted with the Department of Pediatric Gastroenterology due to his history of intractable ascites for the last 4 months. According to his brief medical report from Sudan, he was in a good state of health until he was 2 years old. He presented to a local hospital in Sudan 18 months ago with complaints of 
fever and irritability. Antimalarial medications were empirically given due to the high incidence of malaria in Sudan. In the following 6 months, increase of the head circumference and neurological deterioration such as loss of abilities to sit up, walk, and swallow occurred gradually. Ten months ago, the patient underwent right-sided ventriculoperitoneal (VP) shunt surgery to relieve his hydrocephalus but the intracranial mass excision could not be performed concurrently. His neurologic deterioration progressed and ascites developed. Serial paracentesis during the last 4 months in Sudan did not improve his symptoms and he was referred to our hospital's Department of Neurosurgery and admitted to Intensive Care Unit. At admission, he was lethargic. Vital parameters showed no fever but tachypnea, tachycardia and hypotension. On physical examination, macrocephaly, setting-sun eye phenomenon, severe malnutrition with muscle wasting, dyspnea, severe abdominal distention, umbilical hernia as well as the findings of dullness on percussion and transmitted fluid wave were determined (Fig. 1). Liver and spleen could not be balloted. No other physical stigma revealing chronic liver disease were observed. Despite his oral automatisms and deterioration of consciousness, cranial nerve functions and deep tendon reflexes showed no abnormalities. Pathologic reflexes were negative.

In laboratory analysis, transaminases (ALT: $15 \mathrm{U} / \mathrm{L}, \mathrm{AST}: 23 \mathrm{U} / \mathrm{L})$, tests for liver synthesis functions (albumin: $4.15 \mathrm{~g} / \mathrm{dL}$, prothrombin time: 13.7 seconds, INR: 1.12, ammonia: 52 $\mathrm{ug} / \mathrm{dL}$ ) and renal functions were normal. Viral serology results for Hepatitis B and C, Toxoplasma, Rubella, Cytomegalovirus, Herpes viruses excluded active or chronic infection. Further work up for autoimmune liver disease, Wilson's disease and other metabolic diseases were negative. Neither findings of chronic liver disease and renal disease nor a shunt related pseudocysts were determined on the abdominal ultrasonography. The only positive finding was massive ascites. Portal and hepatic vein flow parameters excluded portal hypertension and
Budd-Chiari syndrome. Echocardiography was normal.

Biochemical as well as microbiological analysis of cerebrospinal fluid (CSF) and ascites were evaluated. Despite the high level of CSF protein (1723 mg/L), glucose level of CSF was normal (CSF glucose: $116 \mathrm{mg} / \mathrm{dL}$ and serum glucose: $105 \mathrm{mg} / \mathrm{dL}$ ). The gross appearance of ascitic fluid was so clear that it resembled the CSF and according to laboratory analysis it was classified as transudate. Microbiological and cytological studies of CSF were negative. ZiehlNeelsen staining of the ascites was negative. No malignant cells, bacteria or parasites were determined in cytological evaluation. Cultures of ascites were negative for bacterial agents including tuberculosis. The cranial magnetic resonance imaging identified significant



Fig. 1. The patient before externalization of the ventriculoperitoneal shunt. 
communicant hydrocephaly, compression of cerebral and cerebellar parenchyma and a multi-lobulated hypervascular mass $(49 \times 49 \times 53$ $\mathrm{mm}$ ) located in the left lateral ventricle. Excessive amount of CSF produced by a tumor and drained into the abdomen via V-P shunt was hypothesized to be the underlying reason. Hence, shunt externalization was suggested to the neurosurgeon in order to improve clinical situation before tumor surgery. In the first operation, the surgeon externalized the abdominal end of V-P shunt from the neck and performed large volume paracentesis. Ten days later, the V-P shunt was totally removed and external ventricular drainage (EVD) was placed. Approximately 1000-1200-mL/day of CSF was drained from EVD and no ascites developed afterwards (Fig. 2). Three weeks later the intraventricular tumor was totally excised. The pathologic assessment revealed choroid plexus $(\mathrm{CP})$ papilloma. In the following

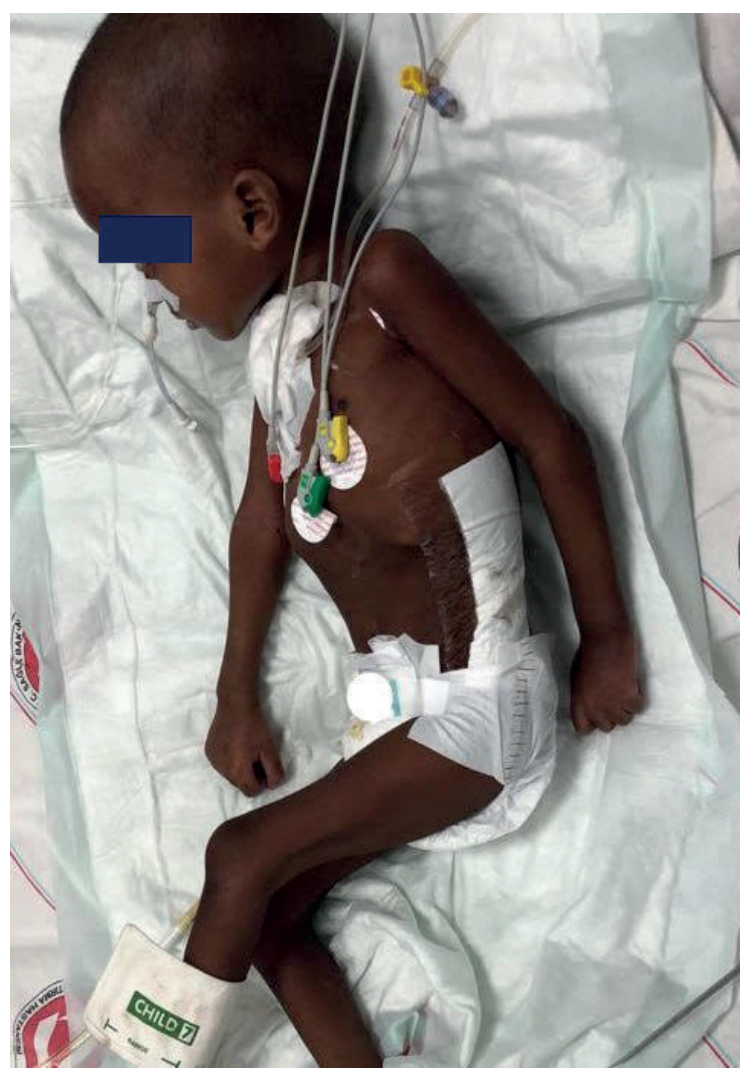

Fig. 2. The patient after choroid plexus excision surgery. days, the patient started to swallow liquids effectively and was discharged on the $40^{\text {th }}$ day of the curative surgery after sufficient weight gain.

Written informed consent regarding clinical data and any accompanying photographs was obtained from the patient's parents.

\section{Discussion}

Ventriculoperitoneal shunt surgery was developed to manage hydrocephalus. ${ }^{4,5,6}$ Due to its safety, convenience, and lower systemic infection risk, the procedure that is targeting to benefit from the absorption function of the peritoneum, diverting the CSF into the abdomen by a V-P shunt, is preferred. ${ }^{5}$ However, several complications of V-P shunts from the first day to $12^{\text {th }}$ year of the surgery have been reported. ${ }^{3}$ Infection, malfunction and occlusion of the shunt are the most common complications. Shunt migration into the thorax (hydrothorax) and scrotum (hydrocele), shunt pseudocyst, intracranial haemotoma, intestinal perforation and shunt/CSF ascites are rarely encountered after V-P shunts. ${ }^{3,5}$

This patient was referred to our hospital for the management of his two problems; an intracranial tumor and ascites, which is a more significant problem that enabled us to reach the main diagnosis. After exclusion of other common non-cirrhotic ascites' reasons (nephrotic syndrome, Budd-Chiari syndrome, tuberculosis, and cardiac failure), the possibility of CSF overproduction was considered in the case of intracranial tumor and V-P shunt implantation.

Reasonable amount of CSF in the abdomen is expected after V-P shunts. However, CSF ascites is an infrequent complication of $\mathrm{V}-\mathrm{P}$ shunt. While the pathophysiology is still not clear, two possible mechanisms accused for CSF ascites formation are exaggerated secretion of CSF from a tumor and reduced absorption of CSF due to peritoneal infection and/or inflammation. ${ }^{3}$ 
Other contributing factors and their possible mechanisms for CSF ascites are listed as;

1) Peritoneal seeding of a malignant tumor via increase in:

- CSF protein level and oncotic pressure within the peritoneal cavity

- Peritoneal permeability by over secretion of vascular permeability factor

2) Chronic infection such as tuberculosis meningitis via increase in CSF protein level

3) Abdominal surgery or repetitive shunt revisions via formation of adhesions and inflammation

4) Chemotherapy via inflammation related malabsorption

5) Immunizations via immune response related inflammation

6) Catheter rejection via eosinophilic inflammation. ${ }^{3,4,6}$

Nonetheless, some cases still remain idiopathic. ${ }^{4}$

Intracranial pathologies such as $\mathrm{CP}$ tumor, craniopharyngioma, and optic-hypothalamic glioma have been reported to result in cerebrospinal ascites after V-P shunt implantation. ${ }^{5,6}$

Choroid plexus tumor of the central nervous system, a spectrum disorder based on tumor grading, covers $\mathrm{CP}$ hyperplasia, $\mathrm{CP}$ papilloma, and CP carcinoma. ${ }^{7}$ Choroid plexus papillomas are benign tumors located mostly in lateral ventricles and that can be cured by surgical resection. ${ }^{8}$ They mainly present in the first 10 years of life, especially in the first two years, with signs of increased intracranial pressure and hydrocephalus. ${ }^{7}$ Choroid plexus is made of tufts of villi producing 100-150 mL of CSF daily in healthy adults and nearly half of that volume in children. ${ }^{4}$ Besides, CP papilloma produces huge amount of CSF such as 400-5000 mL daily. ${ }^{9}$
After we implanted the EVD, 1000-1200 mL of CSF was discharged daily, and no longer intraabdominal fluid accumulated as a supporter of the CSF overproduction hypothesis.

On the top of the differential diagnosis list, there is $\mathrm{CP}$ hyperplasia, a low-grade form of the $\mathrm{CP}$ tumor. Choroid plexus hyperplasia (diffuse villous hyperplasia of the choroid plexus) is defined as the bilateral symmetrical enlargement of the $\mathrm{CP}$ with a normal choroidal morphology and distinguished from $\mathrm{CP}$ papilloma based on the pathologic assessment. ${ }^{7}$ Infection-related pseudocyst of the shunt should also be excluded in case of V-P shunt and excessive abdominal fluid association. ${ }^{5}$

The literature about the treatment for CSF ascites reveals that many of the cases related to various etiologies other than infections have resolved by diverting the shunt into the atrium (ventriculoatrial shunt) ${ }^{4}$ or rarely into the gallbladder. ${ }^{10}$ Ventriculopleural shunt is not advised due to the structural similarity of the pleura with the peritoneum in order to prevent hydrothorax. In case of infection, EVD is advised besides the aggressive antibiotherapy. ${ }^{5}$ The optimal treatment for $\mathrm{CP}$ papilloma is surgical resection but the vascular structure of the tumor makes it challenging. ${ }^{7}$

In conclusion, massive sterile ascites is a rare complication of V-P shunt. The clinician should be suspicious about an etiology that affects the balance between absorption and secretion of CSF in case of intractable ascites accompanying a V-P shunt. The macroscopic feature of the ascitic fluid such as clarity may be the first clue. Differential diagnosis of ascites should be done carefully because the management of the various etiologies is different. Ruling out infections, pseudocyst and malignancy by imaging techniques and ascitic fluid analyses should be the first steps of the CSF ascites algorithm. The shunt externalization gives the advantage of relieving symptoms besides identifying the source of ascites. 


\section{REFERENCES}

1. Blackburn SC, Stanton MP. Anatomy and physiology of the peritoneum. Semin Pediatr Surg 2014; 23: 326330.

2. Van Baal JOAM, Van de Vijver KK, Nieuwland R, et al. The histophysiology and pathophysiology of the peritoneum. Tissue Cell 2017; 49: 95-105.

3. Yukinaka M, Nomura M, Mitani $T$, et al. Cerebrospinal ascites developed 3 years after ventriculoperitoneal shunting in a hydrocephalic patient. Intern Med 1998; 37: 638-641.

4. Diluna ML, Johnson MH, Bi WL, Chiang VL, Duncan CC. Sterile ascites from a ventriculoperitoneal shunt: a case report and review of the literature. Childs Nerv Syst 2006; 22: 1187-1193.

5. Popa F, Grigorean VT, Onose G, Popescu M, Strambu V, Sandu AM. Laparoscopic treatment of abdominal complications following ventriculoperitoneal shunt. J Med Life 2009; 2: 426-436.
6. West GA, Berger MS, Geyer JR. Childhood optic pathway tumors associated with ascites following ventriculoperitoneal shunt placement. Pediatr Neurosurg 1994; 21: 254-258.

7. Bahar M, Hashem H, Tekautz T, et al. Choroid plexus tumors in adult and pediatric populations: the Cleveland Clinic and University Hospitals experience. J Neurooncol 2017; 132: 427-432.

8. Mohindra S, Savardekar A. Management problems in a case of third ventricular choroid plexus papilloma. J Pediatr Neurosci 2012; 7: 40-42.

9. Hori YS, Nagakita K, Ebisudani Y, Aoi M, Shinno Y, Fukuhara T. Choroid plexus hyperplasia with intractable ascites and a resulting communicating hydrocele following shunt operation for hydrocephalus. Pediatr Neurosurg 2018; 53: 407-412.

10. Olavarria G, Reitman AJ, Goldman S, Tomita T. Post-shunt ascites in infants with optic chiasmal hypothalamic astrocytoma: role of ventricular gallbladder shunt. Childs Nerv Syst 2005; 21: 382384. 